\title{
Hypothesis: Free SARS-CoV-2 spike protein S1 particles may act as a factor of COVID-19 pathogenesis.
}

Andrey V. Letarov ${ }^{1,2, *}$ Vladislav V. Babenko ${ }^{3}$

${ }^{1}$ Winogradsky Institute of Microbiology, RC Biotechnology RAS, Moscow, Russia

${ }^{2}$ Faculty of Biology, Lomonosov Moscow State University, Moscow, Russia

${ }^{3}$ Federal Research and Clinical Center of Physical-Chemical Medicine, Federal Medical Biological Agency, Moscow, Russia

*Corresponding author. letarov@gmail.com, Pr. 60-letiya Oktyabrya 7 build. 2. 117312, Moscow, Russia

\begin{abstract}
The disbalance of the renin-angiotensin system was suggested to play an important role in the pathogenesis of the COVID-19 disease. Previously it has been shown that ACE2 expression in downregulated in the murine model in response to SARS-CoV infection and may be also induced by the recombinant spike protein alone. We hypothesize that the soluble SARS-CoV-2 spike protein S1 subunits shed from the infected cells and from the virions in vivo may bind to the ACE2 receptor and trigger ACE2 downregulation. Decreased ACE2 activity on the background of the constant or increased ACE activity in the lungs may lead to the prevalence of angiotensin II effects over angiotensin(1-7) connected to increased thrombosis, inflammation and pulmonary damage.
\end{abstract}

Keywords: SARS-CoV-2, renin-angiotensin system, Spike protein S1 subunit, COVID-19 pathogenesis.

The pandemic of COVID-19 disease that caused already hundreds of thousands of fatalities worldwide is caused by the coronavirus SARS-CoV-2 that is most probably transited from an animal host to humans in late $2019^{1}$. The virus is highly related to the bat Coronovirus RaTG13, however, the existence of a transient host (probably a pangolin) was suggested ${ }^{2}$. The adaptation of SARS-CoV-2 virus to human host is believed to be mostly due to acquisition of the novel sequence of the tail spike (S) protein receptor binding domain (RBD) that efficiently recognizes human angiotensin converting enzyme 2 (ACE2). Although a related SARS-CoV coronavirus also recognizes ACE2 as its cellular receptor ${ }^{1}$, only 8 out of 14 critical a.a. residues involved into the interaction of the ACE2 and RBD are conserved between SARS-CoV and SARSCoV-2 viruses ${ }^{2}$.

The coronavirus entry into the host cell requires cleavage of the $S$ protein by the host proteases at the junction between $\mathrm{S} 1$ and $\mathrm{S} 2$ subunits ${ }^{1}$. This processing can take place after the virion attachment by the cell surface protease TMPRSS2 or in the lysosomal compartment after the internalization of the virus ${ }^{1,3}$. The proteolytic processing of S protein allows S1 subunit to dissociate to trigger the $\mathrm{S} 2$ subunit rearrangement to extended post-fusion conformation required for initiation of the fusion of the viral and lysosomal membranes ${ }^{4}$.

In some coronaviruses, however, the $S$ protein processing may take place during the virion assembly in the Goldgi compartment. Such processing requires furin protease recognition site to be present at the S1 and S2 subunits junction ${ }^{3}$. In SARS-CoV-2 the insertion of 4 a.a. sequence PRRA after the a.a. 675 in S protein that lead to the formation of the furin cleavage site RRAR ${ }^{2}$. This leads to efficient processing of the $S$ protein in the virus producing cell ${ }^{3,5}$. The 
pre-processing of the spike protein before the virus release makes the infection more efficient ${ }^{3}$ and potentially may allow part of the virus particles to penetrate into the host cells directly at the plasma membrane without entering to the lysosomal compartment ${ }^{6}$. More efficient host cell entry taken together with higher affinity of its RBD (compared to RBD of SARS-CoV that lacks efficient furin processing site) may compensate SARS-CoV-2 for decreased availability of the RBDs for ACE2-receptor binding ${ }^{3}$. Indeed, on most of the SARS-CoV-2 S protein trimers two out of three RBDs remain in closed conformation in which they are shielded from both host immunity factors (e.g. antibodies) and from receptor recognition, while in SARS-CoV virus all three RBDs are in "open" conformation on most of the spikes ${ }^{7}$.

As in any virus pneumonia, the pathology of COVID-19, as well of SARS, is believed to be mainly due to the virus killing of the lung epithelial cells, and, even to larger extent, due to immunemediated mechanisms ${ }^{1,8}$.

However, the recognition of ACE2 as the receptor by both SARS-CoV and SARS-CoV-2 raised a hypothesis that in addition to the aforementioned mechanisms (that may be further enhanced by a secondary bacterial infection) the pathology of COVID-19 and of SARS may in large extent rely on the virus-induced disbalance of the renin-angiotensin system (RAS) ${ }^{9,10}$.

ACE2 is a cell-surface metalloprotease (carboxypeptidase) that converts angiotensin I decapeptide into angiotensin ${ }_{1-9}$ octapeptide, in contrast to ACE enzyme, that produced out of angiotensin I the physiologically active angiotensin II octapeptide. Angiotensin 1-9 can be converted by ACE enzyme into angiotensin ${ }_{1-7}$ heptapetide. Angiotensin II also may be processed into angiotensin ${ }_{1-7}$ by ACE2 ${ }^{9,10}$. The physiological activity of angiotensin II leads to vasoconstriction and increased arterial pressure but it also promotes inflammation reactions, blood coagulation and thrombosis, fibrosis capillary permeability and edema. Angiotensin ${ }_{1-7}$ has basically opposite effect decreasing inflammation, thrombosis, fibrosis and causes vasodilatation ${ }^{9,10}$. Therefore, upregulation of ACE and/or downregulation of ACE2 lead to increased pulmonary damage.

It has been demonstrated that SARS-CoV infection decreases ACE2 expression in murine model 9-11 suggested that virus attachment to ACE2 molecules lead to their removal from the cell surface via co-endocytosis with the virus thus diminishing the ACE2 activity. This leads to the imbalance between angiotensin II and angiotensin ${ }_{1-7}$ in the lung tissue and to increases thrombosis and pulmonary damage ${ }^{9,10}$.

From our point of view, the direct mechanical removal of the ACE2 molecules by the virion attachment is unlikely to contribute significantly to the overall activity of the enzyme in the lungs. Such an effect would require simultaneous virus attachment to large fraction of the ACE2 producing cells (such as avleocytes II). We believe that at that high virus load the direct damage of the epithelial cells would not be compatible with the patient survival, though the reported mortality even in severe COVID-19 cases is moderate ${ }^{1,8}$. Interestingly, in ${ }^{11}$ study the reduction of ACE2 in mice could be induced not only by SARS-CoV infection but also by the recombinant SARS-CoV spike protein. The mice pre-treated (i.p.) with this spike protein did not show significant pathology but if these animals were acid instilled, the spike protein pre-treatment lead to increased severity of the lung damage ${ }^{11}$.

Therefore, the spike protein if present in significant molar excess in respect to the viral particles may in fact mediate the down regulation of ACE2 and RAS imbalance. It has been shown that $S$ proteins of murine coronaviruses and of SARS-CoV are indeed delivered onto the cell surface, presumably, as a side product of the virus assembly and release process. These molecules may induce some physiological effect such as micropinocytosis and/or membrane fusion of the neighbor cells ${ }^{6}$, however $S$ protein been a membrane protein, remains attached to the infected cell membrane. It worth to mention that $\mathrm{S}$ protein is also present on the viral particles released from the infected cells into the medium. 
It has been recently demonstrated by direct cryo-electron and negative-stain electron microscopy ${ }^{12}$ that on the in vitro cultured SARS-CoV-2 particles retaining their infectivity most of $S$ protein trimers are in the post-fusion conformation. In the other words, the S1 subunits dissociation took place before the actual receptor binding (apparently the biological activity of these virions was assured by the remaining fraction of $S$ trimers that did not yet shot in vain). Although in the conditions of the study, the S1 subunits dissociation could be triggered by the conditions during the gradient centrifugation used to purify the virus ${ }^{12}$. Nevertheless, this result demonstrates that in SARS-CoV-2 processed spikes S1 subunit dissociation can be triggered by relatively mild conditions that are compatible with the virus viability, or occur spontaneously. We hypothesize that substantial amount of free soluble S1 subunits may be shed from the infected cells and virions (Fig 1).

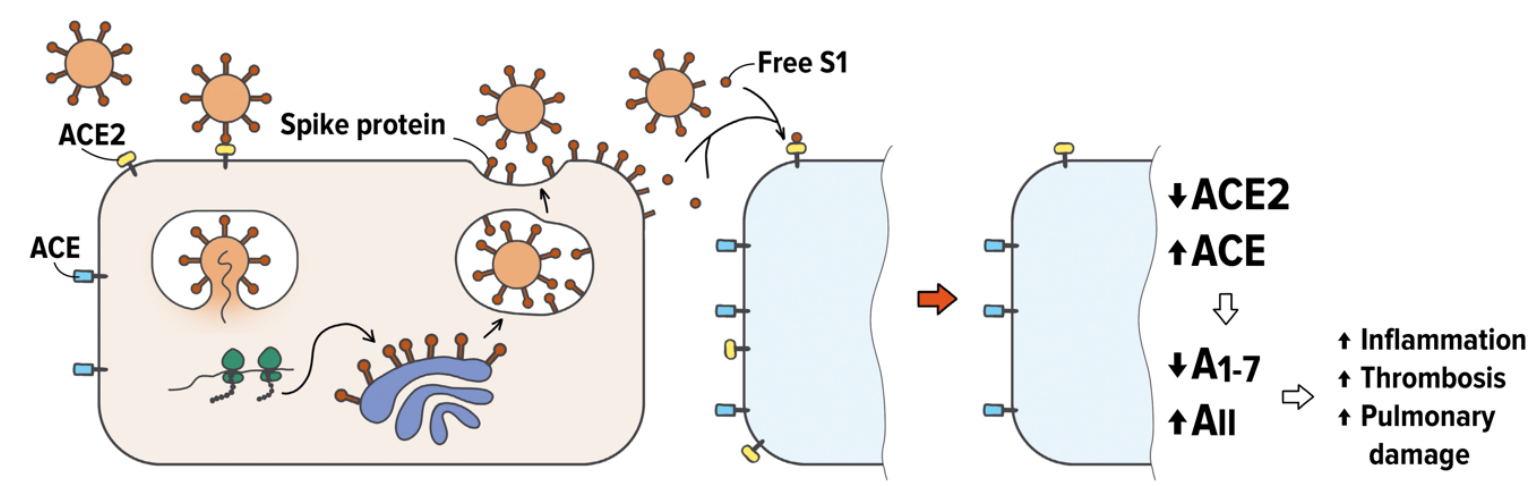

Figure 1.

Hypothesis of involvement of free S1 subunits of SARS-CoV-2 spike protein into COVID-19 induced

Binding to the receptor via their intact RBDs these molecules may induce ACE2 downregulation and the downstream deleterious effects as it was suggested by Gheblawi et al. and Verdecchia et al. ${ }^{9,10}$.

If our hypothesis turns true, free S1 molecules may represent a target for COVID-19 therapy or prevention. After the separation of S1 subunit from S2 stem the potential epitopes free of the glycans shielding the external surface of the complete $S$ protein ${ }^{13}$ become exposed. So the immunization by the recombinant proteins that would elicit the antibodies response against these conserved and unprotected epitopes may lead to the sequestration of free S1 molecules to immune complexes and their elimination thus reducing the probablility of severe COVID-19 pneumonia.

\section{Acknowledgements.}

Authors a grateful to Ms. Ksenya Sayfulina from Moscow State University of Psychology and Education for the help with the figure creation.

\section{References}

1. Chen, Y., Liu, Q. \& Guo, D. Emerging coronaviruses: Genome structure, replication, and pathogenesis. J. Med. Virol. 92, 418-423 (2020).

2. Zhang, T., Wu, Q. \& Zhang, Z. Probable Pangolin Origin of SARS-CoV-2 Associated with the COVID-19 Outbreak. Curr. Biol. 30, 1346-1351.e2 (2020).

3. Shang, J. et al. Cell entry mechanisms of SARS-CoV-2. Proc. Natl. Acad. Sci. U. S. A. (2020) doi:10.1073/pnas.2003138117. 
4. Walls, A. C. et al. Tectonic conformational changes of a coronavirus spike glycoprotein promote membrane fusion. Proc. Natl. Acad. Sci. U. S. A. 114, 11157-11162 (2017).

5. Markus Hoffmann, H. K.-W. S. P. A multibasic cleavage site in the spike protein of SARSCoV-2 is essential for infection of human lung cells. Cell Press (2020) doi:10.1016/j.molcel.2020.04.022.

6. Freeman, M. C., Peek, C. T., Becker, M. M., Smith, E. C. \& Denison, M. R. Coronaviruses induce entry-independent, Continuous macropinocytosis. MBio 5, 1-10 (2014).

7. Wrapp, D. et al. Cryo-EM structure of the 2019-nCoV spike in the prefusion conformation. Science (80-. ). 367, 1260-1263 (2020).

8. Matricardi, P. M., Dal Negro, R. W. \& Nisini, R. The first, holistic immunological model of COVID-19: implications for prevention, diagnosis, and public health measures. Pediatr. Allergy Immunol. pai.13271 (2020) doi:10.1111/pai.13271.

9. Gheblawi, M. et al. Angiotensin Converting Enzyme 2: SARS-CoV-2 Receptor and Regulator of the Renin-Angiotensin System. Circ. Res. (2020) doi:10.1161/circresaha.120.317015.

10. Verdecchia, P., Cavallini, C., Spanevello, A. \& Angeli, F. The pivotal link between ACE2 deficiency and SARS-CoV-2 infection. European Journal of Internal Medicine vol. 0 (2020).

11. Kuba, K. et al. A crucial role of angiotensin converting enzyme 2 (ACE2) in SARS coronavirus-induced lung injury. Nat. Med. 11, 875-879 (2005).

12. Liu, C. et al. Viral Architecture of SARS-CoV-2 with Post-Fusion Spike Revealed by CryoEM. bioRxiv 2020.03.02.972927 (2020) doi:10.1101/2020.03.02.972927.

13. Watanabe, Y., Allen, J. D., Wrapp, D., McLellan, J. S. \& Crispin, M. Site-specific glycan analysis of the SARS-CoV-2 spike. Science (2020) doi:10.1126/science.abb9983. 